\title{
Reviews
}

\section{The use of cleavage site specific antibodies to delineate protein processing and breakdown pathways}

\author{
J S Mort, D J Buttle
}

\begin{abstract}
The hydrolysis of peptide bonds is an integral part of most physiological and pathological processes, yet knowledge is often lacking as to which peptide bonds are cleaved, in which protein substrates, in which order, and by which proteolytic enzymes. An increase in our understanding of these processes will enhance understanding of the pathogenesis underlying many diseases and might aid in the recognition of new targets for therapeutic intervention. This article reviews the development, design, and use of antibodies for the detection of specific peptide bond cleavage events, and describes how the application of such antibodies can increase our understanding of the roles played by proteolytic enzymes in physiology and pathology.

(F Clin Pathol: Mol Pathol 1999;52:11-18)
\end{abstract}

Keywords: protein synthesis; proteolysis; cleavage site specific antibodies; aggregan; collagen

Protein turnover-the continuous synthesis, modification, and breakdown of proteins - is a feature of all biological systems. In healthy adult tissue, the synthesis and breakdown of proteins is maintained in a stable equilibrium. However, during development and growth, and in many pathological situations, this "equation" becomes unbalanced. Many disease states are characterised by altered rates of protein synthesis and modification, or increased rates of protein breakdown. Despite their undoubted involvement in development and in pathology, the precise mechanisms of protein modification and degradation remain poorly understood.

There are two major pathways of protein synthesis in eukaryotes, one usually reserved for the production of cytosolic proteins and one for secreted proteins, where "secretion" includes transportation into membrane bound vesicles or attachment to the external surface of the cell, as well as the discharge of newly synthesised soluble proteins into the extracellular environment. In general, the synthesis of cytosolic proteins does not involve protein cleavage. However, proteins destined for other compartments are modified by peptide bond hydrolysis as part of their processing and trafficking mechanism. Proteins that are intended for incorporation into the mitochondria and are synthesised on ribosomes outside this organelle require cleavage of leader peptides for entry into the different compartments of the mitochondrion. Most other secreted proteins are synthesised on the rough endoplasmic reticulum as "preproproteins". A specific peptide bond cleavage takes place while the nascent polypeptide is still attached to the endoplasmic reticulum, resulting in the removal of the signal peptide, this component having fulfilled its role by directing the newly synthesised protein out of the cytoplasm. Further proteolytic modification often occurs in the Golgi apparatus, where the propeptide is removed, and the "mature" protein is secreted, stored in granules, or attached to the outer surface of the cell via a transmembrane region or a glycosylphosphatidylinositol linkage. As always, there are exceptions to these general pathways and the maturation of the protein might continue outside the cell. For example, procollagen is secreted rather than being processed in the Golgi. Collagen fibril formation then occurs extracellularly and is dependent on the removal of the C-terminal propeptide, which is accomplished by the action of a specific proteolytic enzyme. ${ }^{1}$

In addition to proteolytic processes involved in protein synthesis and trafficking, there are many post-translational protein cleavage steps that are required to unveil or, under some circumstances, alter the biological activity of a protein. Most biologically active peptides, such as neuropeptides and peptide hormones, are activated by the proteolytic cleavage of a precursor molecule, and most secreted proteolytic enzymes are themselves activated by proteolytic removal of a propeptide. It is at this level that the exquisite control exerted by peptide bond cleavage can be seen most readily. Examples of such processes occurring outside the cell include fibrin clot formation and removal, complement activation, regulation of blood pressure, and activation of digestive enzymes, whereas antigen processing, apoptosis, and viral particle assembly are examples of
Accepted for publication 5 November 1998 

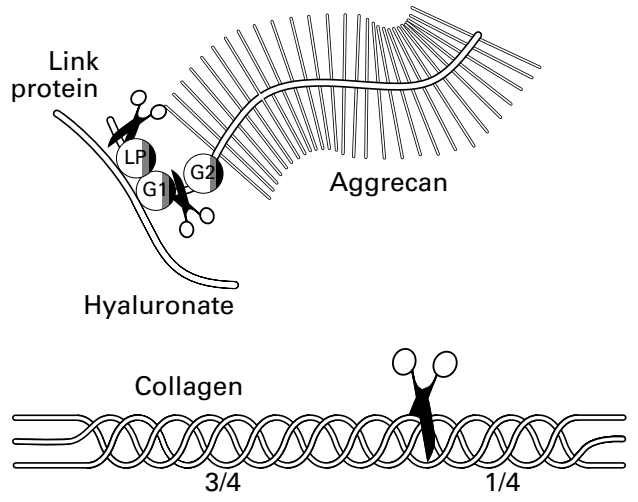

Figure 1 Schematic representation of the three connective tissue molecules that are the major components of articular cartilage and that have been studied by the cleavage site specific antibody approach. Specific cleavage sites have been identified on link protein (LP), aggrecan, and collagen.

intracellular proteolytic processing. Not surprisingly, many of these processes are controlled by proteolytic enzymes displaying a high degree of discrimination with regard to the peptide bonds that are cleaved.

Proteins have varied life spans that depend on the developmental or pathological status of their surroundings, but at some point they are all broken down. The breakdown process is well controlled and, like the preproprotein and proprotein processing steps that occurred earlier, it may be initiated by the cleavage of specific peptide bonds through the action of proteolytic enzymes. Intracellular proteins are either targeted for breakdown by ubiquitination and degraded by the proteasome, a multi-

\section{G1.... DFVDI PEN F GVGGF....G2}

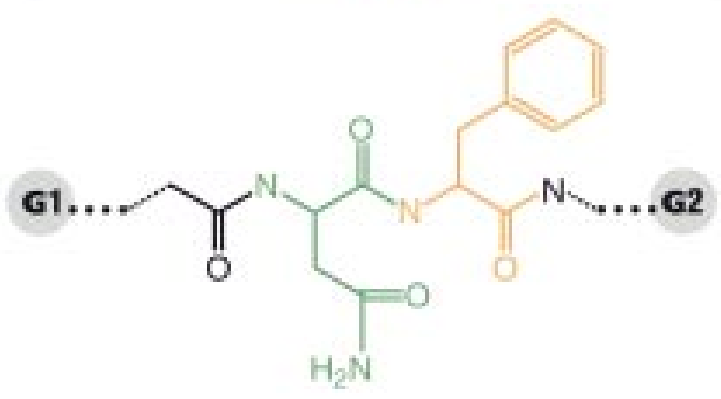

$$
\text { G1.... D F V D I P E N }
$$

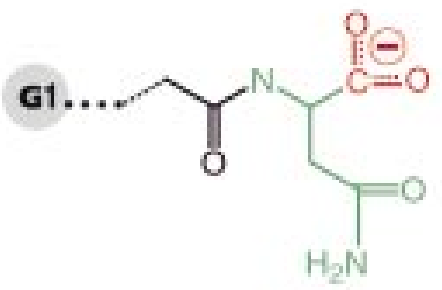

Figure 2 Representation of the generation of cleavage site specific epitopes in the interglobular region of aggrecan. The intact protein is cleaved by various proteases to generate the new epitope "...VDIPEN" with the introduction of a C-terminal negative charge (red) and "FFGVG..." with the introduction of an N-terminal positive charge (blue). subunit cytoplasmic protein degrading complex, ${ }^{2}$ or taken up into lysosomes (a process termed heterophagy) and broken down there. Extracellular proteins may be reclaimed by the cell (endocytosis) and broken down in lysosomes, or their breakdown may occur extracellularly.

Examples abound of the inappropriate action of proteolytic enzymes in pathology, and include the cleavage of the amyloid precursor protein to form amyloidogenic fragments in Alzheimer's disease, ${ }^{3}$ inappropriate activation of trypsinogen in acute pancreatitis, ${ }^{4}$ and increased rates of breakdown of extracellular matrix components in glomerulonephritis, ${ }^{5}$ and in tumour growth and metastasis. ${ }^{6}$ In the latter cases, the breakdown of the extracellular matrix allows both the growth of the primary tumour and extravasation and intravasation of metastasising cells. In arthritis, the breakdown of the cartilage matrix results in joint malfunction and disability, ${ }^{7}$ and diseases such as osteoporosis and Paget's disease result from the increased breakdown of the protein constituents of bone. ${ }^{8}$ In all inflammatory situations there is an increased breakdown and remodelling of extracellular matrix components. Included within this category are a multitude of disease processes, such as cirrhosis of the liver and lung emphysema. ${ }^{9} 10$

Thus, it is well established that the actions of proteolytic enzymes are involved in many aspects of human physiology and pathology. Yet our understanding of the processes that are occurring is still rudimentary. In many cases we do not know which protein constituents are being inappropriately modified or broken down, or which peptide bonds are being cleaved by which proteolytic enzymes. A greater insight into the processes of protein modification and breakdown will help us to develop future treatments for many disease processes. In our review we discuss a methodological approach aimed at identifying specific peptide bond cleavages, in particular using examples from the degradation of extracellular matrix molecules present in articular cartilage (fig 1). Much can be learned about the mechanism of protein breakdown from knowledge of the peptide bond(s) being cleaved because these represent a fingerprint left by the proteolytic enzyme mediating those particular cleavages. Once identified, these enzymes might be appropriate targets for therapeutic intervention. In addition, because this methodology allows the localisation of a particular proteolytic event, it adds to our knowledge and understanding of the mechanisms underlying various pathologies.

\section{Cleavage site specific antibodies: the} meaning of the term

Proteolytic enzymes attack their substrates using two approaches. Exopeptidases remove single residues or dipeptides sequentially from either the $\mathrm{N}$-terminus or C-terminus depending on the specificity of the particular protease, whereas endopeptidases make one or more cleavages within the protein to break it down into smaller polypeptide fragments. A natural 
consequence of protein cleavage is the generation of a new N-terminus if the cleavage is carried out by an aminopeptidase, a new C-terminus if the hydrolysing enzyme is a carboxypeptidase, or novel N-termini and C-termini if an endopeptidase is involved. These termini are antigenically distinct from the same sequence present in the intact protein. Therefore, it is possible to generate antibodies that only recognise a peptide sequence if there is a primary amino group at the N-terminus or a carboxyl group at the C-terminus; the same sequence in the intact polypeptide is not recognised (fig 2). The general term "antineoepitope antibody" has been used to describe such antibodies. ${ }^{11}$ However, this name has also been used to describe antibodies that react with novel epitopes appearing on cells at certain stages of differentiation or transformation. ${ }^{12}$ Thus, we propose the term "cleavage site specific antibodies" to describe antibodies that react exclusively with a cleavage product following the action of a proteolytic enzyme. Therefore, these antibodies are distinct from those that react with a previously hidden epitope which is exposed when the protein is subjected to limited proteolysis, ${ }^{13}$ or antibodies that only react with a protein in a certain conformation ${ }^{14}$; none of those types of antibody is dependent on a primary amino or carboxyl group at the terminus of a protein or peptide for reactivity.

\section{Mechanism}

The experimental evidence provided by the use of a large number of cleavage site specific antibodies demonstrates their specificity for terminal epitopes. Although the molecular mechanism underlying this selectivity has not yet been elucidated, structural studies on immunoglobulins and on proteins that exhibit specificity for charged residues allow us to propose a model for these antibodies. The three dimensional structures of several antipeptide antibodies, crystallised as complexes with their peptide antigens, have been determined and these show that the peptide is usually bound in the form of a loop ( $\beta$ bend) with the termini projecting out of the antigen binding site..$^{15}$ Based on such structures, fig 3 is a schematic representation of a cleavage site specific antibody. In this particular case, this antibody recognises the new C-terminus "...VDIPEN" of the aggrecan G1 domain, produced after cleavage of the "N-F" peptide bond. In this model, the specificity for the C-terminal neoepitope ". . VDIPEN" is a consequence of the antigen binding site forming a closed cavity containing a region of positively charged residues. This cavity accommodates the negatively charged C-terminus of the epitope. In proteins where the recognition of a charged residue is crucial for function - for example, in the case of cysteine proteases of the caspase family, where substrate cleavage always occurs after an aspartic acid residue, ${ }^{17}$ the binding pocket is exquisitely designed to accommodate the negative charge on the aspartic acid side chain. We expect that cleavage site specific antibodies also contain

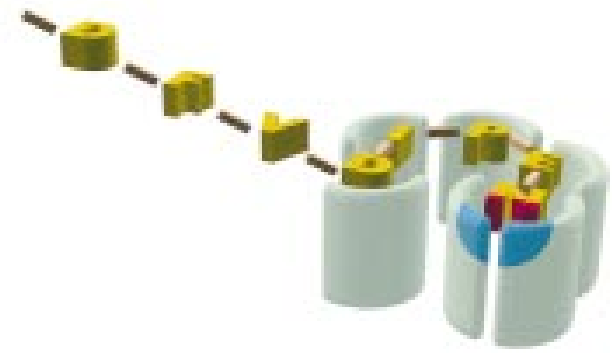

Figure 3 Cartoon representation of the peptide binding region of a cleavage site specific antibody. The strands (green) represent the framework of the Fv region bearing the six antigen binding complementarity determining regions (CDRs) - three each from the heavy and light chains. The peptide antigen is represented by the single letter code for its amino acid residues. Although the antigen binding site is complementary to some aspects of the peptide antigen, some components of the epitope are not very important and do not contribute much to binding. However, of absolutely crucial importance is the presence of a pocket (blue), which accepts the charged group associated with (in this case) the C-terminus of the epitope (red) and seals off the end of the binding site, thereby providing a barrier to prevent binding of the additional residues that would be present on the extended chain of the intact protein from which the epitope was generated.

such a region of charge complementarity forming the basis for their specificity. As also demonstrated in this model, the antibody combining site is believed to accommodate at least five residues of the peptide antigen. However, not all of the side chains make a major contribution to antibody specificity.

\section{Examples}

Although cleavage site specific antibodies are now proving to be important reagents for understanding the fundamental aspects of connective tissue turnover, this technology has a much earlier history. The production of such antibodies using synthetic peptides as the immunising antigen was reported in 1983, when both polyclonal ${ }^{18}$ and monoclonal ${ }^{19}$ antibodies were described that recognised fibrin but not its precursor fibrinogen. Since then, cleavage site specific antibodies have been used to study the proteolytic processing of proteinases-for example, in the case of calpain ${ }^{20}$ and of other enzymes, such as protein kinase C. ${ }^{21}$

Extracellular matrix degradation is an underlying feature of many degenerative diseases, such as arthritis, and the identification of the proteolytic enzymes responsible for the destruction of the different matrix components is an essential step in the development of treatment regimens. An irreversible consequence of the arthritides is loss of articular cartilage. This tissue is composed of a type II collagen network that embeds a matrix of proteoglycan aggregates consisting of the proteoglycan, aggrecan, non-covalently complexed to hyaluronate through the stabilising influence of a small glycoprotein, termed link protein (fig 1)..$^{22}$ Degradation of these components has been shown to be the result of the action of various proteases and the action of specific enzymes is implied by the formation of cleavage site epitopes. ${ }^{23} \mathrm{~A}$ specific example in the case of link protein is shown in fig 4 . 
Link protein is present in human cartilage as three molecular components. The larger $48 \mathrm{kDa}$ and $44 \mathrm{kDa}$ forms (LP1 and LP2, respectively) differ only by the absence of an asparagine linked oligosaccharide located on residue 6 in LP2. The lowest molecular mass form (LP3) is a naturally occurring proteolytic product that increases in abundance during aging. A short fragment (16-23 residues) of the $\mathrm{N}$-terminal region is removed, the specific cleavage point depending on the protease involved. Because of the proximity to the $\mathrm{N}$-terminus of the asparagine linked oligosaccharide, LP3 is a product either of LP1 or $\mathrm{LP} 2 .^{24}$ Matrix metalloproteinases, in particular stromelysin-1, cleave LP1 and LP2 between residues 16 and 17 to produce a cleavage site epitope "IQAENG . . .", whereas other proteases cleave more C-terminally. ${ }^{25}$ The generation of LP3 by stromelysin produced in situ in human articular cartilage is shown in fig 4. Using the monoclonal antibody 8A4, which recognises an internal epitope, the three link protein components can be visualised in a western blot of a human cartilage extract. When active stromelysin- 1 is generated in situ, an increase in the intensity of the LP3 component is seen. When a similar blot is probed with the monoclonal antibody $\mathrm{CH}-3$, which recognises the cleavage site epitope
"IQAENG . ..", ${ }^{23}$ only a single band migrating at the position of LP3 is seen and its intensity increases dramatically under conditions where active stromelysin is generated, indicating the ability of this enzyme to process link protein in cartilage.

Both monoclonal and polyclonal cleavage site specific antibodies have been produced that specifically recognise the new termini generated on cleavage of aggrecan by matrix metalloproteinases (the " . . .VDIPEN" epitope shown in fig $2^{2627}$ and the new $\mathrm{N}$-terminal epitope" FFGVG . .."28) and by a still incompletely characterised activity termed "aggrecanase", which cleaves the aggrecan interglobular region to generate the epitopes “. . .NITEGE" and "ARGSV . ..". ${ }^{26}{ }^{29} 30$ The availability of such reagents has greatly facilitated the study of aggrecan breakdown in normal cartilage and in arthritis. ${ }^{31} 32$

The triple helical nature of type II collagen, the other major component of cartilage, makes this molecule resistant to the action of most proteases. However, three collagenases, members of the matrix metalloproteinase family, take advantage of a position located towards the C-terminus of the molecule, where the triple helix appears to be less rigid. ${ }^{33}{ }^{34}$ Cleavage at this position yields two fragments termed $\mathrm{TC}^{\mathrm{A}}$ and $\mathrm{TC}^{\mathrm{B}}$, or the three quarter and one
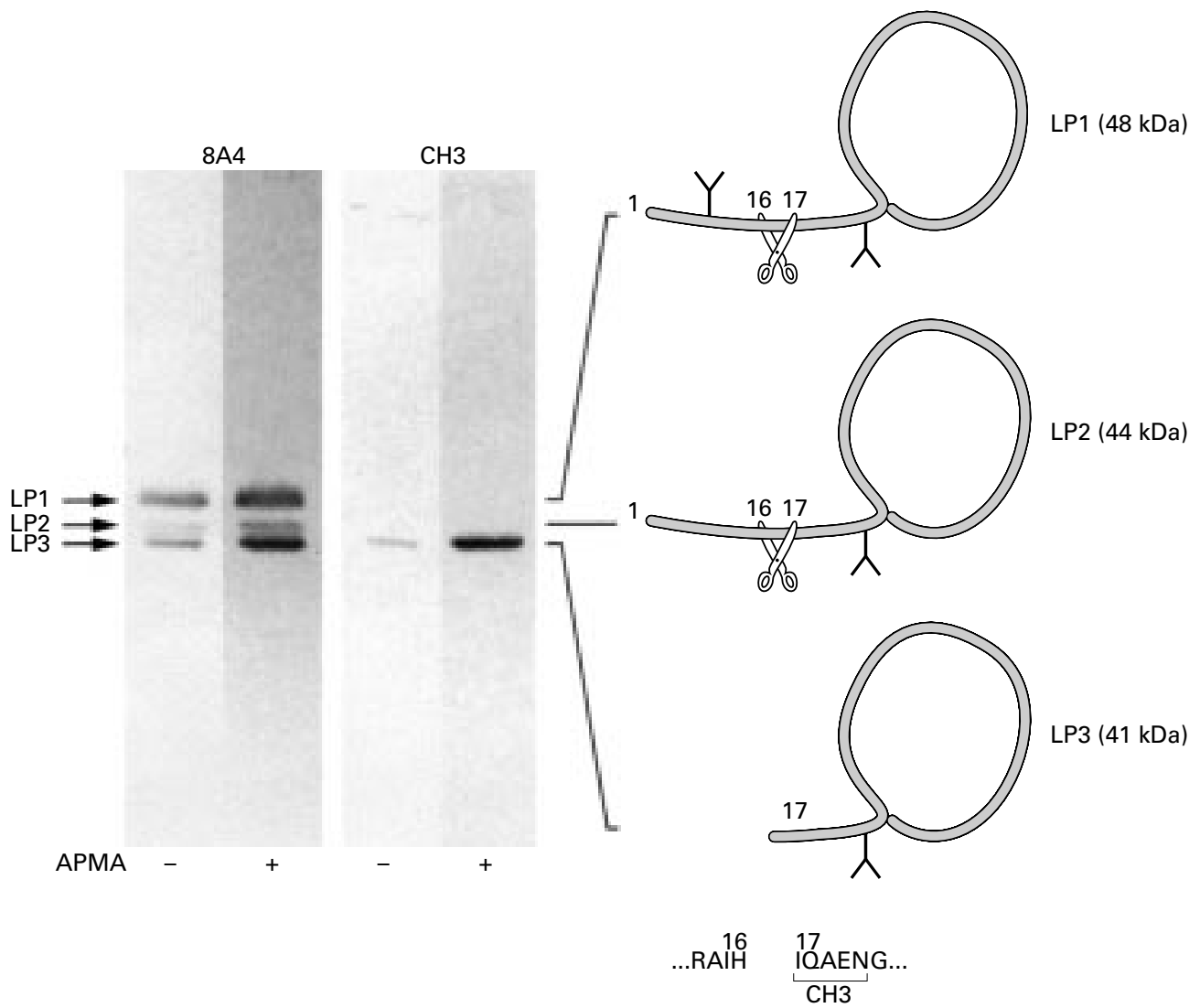

Figure 4 Matrix metalloproteinase production of the link protein neoepitope LP3. Human articular cartilage explants were cultured in the presence of interleukin $1 \beta$, which induces the biosynthesis of prostromelysin-1. This proenzyme was then artificially activated in situ by addition of p-aminomercuric acetate (APMA). The tissue was then extracted with guanidinium chloride and the resulting proteins analysed by polyacrylamide gel electrophoresis in the presence of sodium dodecyl sulphate, followed by electroblotting on to a nitrocellulose membrane. The resulting western blot was probed using a general link protein antiserum (8A4) or a cleavage site specific antiserum (CH-3) specific for the epitope "IQAENG ...". The right hand side of the figure illustrates the three forms of link protein present in cartilage, and the sequences around the cleavage site for matrix metalloproteinases, in particular stromelysin-1, which is recognised by the monoclonal antibody $\mathrm{CH}-3$. The " $\mathrm{Y}$ " shape represents an asparagine linked oligosaccharide. 

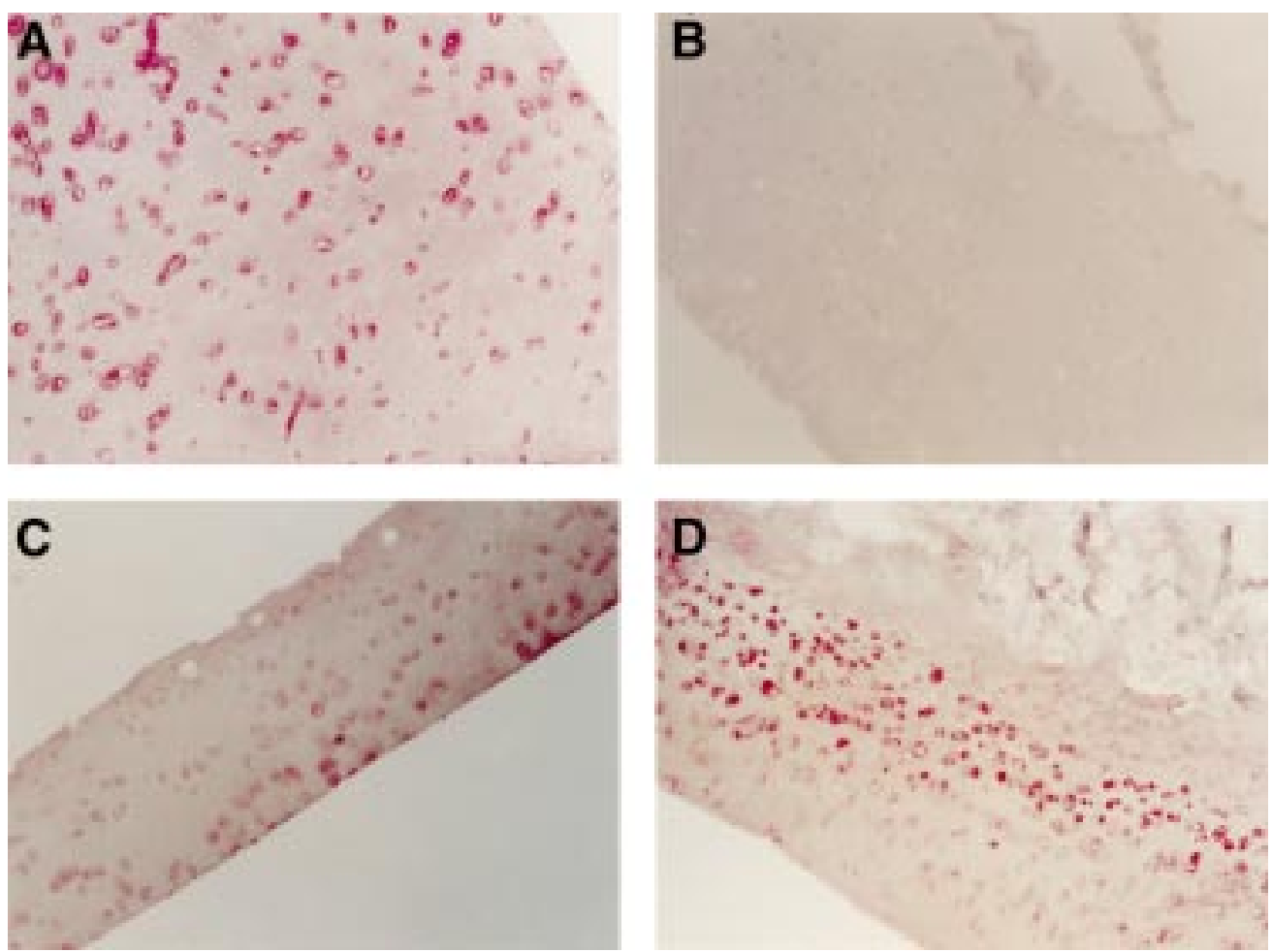

Figure 5 Immunohistochemical analyses of human knee articular cartilage specimens using a rabbit antiserum (MV-1) directed against the $\mathrm{N}$-terminal neoepitope produced by the action of collagenases on type II collagen. The sections are oriented with the articular surface towards the top. Cryosections were stained using the ABC method (Vector laboratories, Burlington, Ontario, Canada), as described previously. ${ }^{36}$ (A) Sample from a 65 year old man with no clinical signs of arthritis. Note the pericellular staining indicative of localised breakdown of type II collagen in the normal cartilage. (B) Section from the same sample as $(A)$ stained using preimmune serum. Sections from patients with arthritis showed a similar absence of staining with preimmune serum. (C) Section from a 63 year old woman with osteoarthritis. Pericellular staining is most intense in the deep zone. There is also diffuse staining of the cartilage matrix remote from the cells in the deep and mid zones. (D) Section from a 54 year old woman with rheumatoid arthritis. In contrast to the osteoarthritic cartilage, intense pericellular staining is seen only in the mid zone, immediately below the region of pannus invasion. (Original magnifications, $\times 10$.)

quarter fragments, respectively (fig 1). As with other connective tissue proteins, this cleavage generates specific novel termini for which cleavage site specific antibodies have been generated. ${ }^{35} 36$

During synthesis and maturation, collagen fibres undergo extensive crosslinking so that, although they may have been damaged as a result of proteolytic attack, the cleavage fragments might remain in the tissue. Immunohistochemical localisation of cleavage site epitopes provides an important method to assess collagen damage in diseases such as arthritis. As illustrated in fig 5, a small amount of cleaved collagen is found pericellularly in normal adult human cartilage and this staining is distributed evenly throughout the matrix. In contrast, osteoarthritic cartilage taken at surgery for arthroplasty showed strong staining in the deep zone, suggesting that late in the disease process collagenases are active in this area. In cartilage taken from patients with rheumatoid arthritis, intense staining was seen in the mid zone immediately below the invading pannus. The differential staining seen in different disease states indicates that collagen degradation does not occur uniformly throughout the tissue. Further investigation of the temporal distribution of this process during disease progression will be of great interest.

\section{Methodology}

Cleavage site specific antibodies can be produced both by the monoclonal or polyclonal route. Whereas the monoclonal approach allows the selection of hybridomas specific for the terminal epitope, it might be expected that polyclonal antibodies would be of limited value because a mixed immunoglobulin population should be produced, not all of which would have the required specificity for the cleavage epitope. However, in reality, terminal epitopes often appear to be dominant so that cleavage site specific sera are produced. If necessary, affinity purification using the immunising peptide can be used to increase the specificity.

Peptides equivalent to the amino acid sequences on either side of the scissile bond are synthesised by standard solid phase chemistry. It is crucial that the peptide contains the appropriate $\alpha$-amino or C-terminal carboxyl group. The addition of a cysteine residue at the opposite terminus of the epitope facilitates coupling to a protein carrier for immunisation. The insertion of one or two glycine spacer residues between this cysteine residue and the epitope allows accessibility of the antigenic peptide without providing additional immunogenic material. The peptide antigens should be quite short, thus reducing the chance of generating antibodies that react with a part of the sequence remote from the terminus. Good 
results have been obtained with peptides 8-11 amino acids in length (including Gly and Cys). The peptide is coupled to a carrier molecule using a bifunctional reagent capable of reacting both with the thiol group of cysteine present on the peptide and amino groups on the carrier protein. Many such reagents are available; however, N-hydroxysuccinimidyl bromoacetate is very useful because the resulting linker is extremely small. ${ }^{37}$ The carrier molecule can be almost any protein but, ideally, it should be highly immunogenic and should not contain amino acid sequences that might lead to antibody crossreactivity in the biological system to be analysed. It should also not contain any free cysteine residues, which could lead to polymerisation of the protein on reaction with the bifunctional reagent. Ovalbumin and keyhole limpet haemocyanin have been used frequently. The carrier molecule is first reacted with the bifunctional reagent, and the derivatised protein separated from unreacted reagent by gel filtration chromatography. The activated carrier protein is then added to the peptide and coupling is allowed to take place. The success of the coupling reaction is conveniently assessed by comparison with the uncoupled carrier molecule on polyacrylamide gel electrophoresis in the presence of sodium dodecyl sulphate (SDS). ${ }^{23}$ When the coupled peptide is one that contains one or more aromatic residue, the degree of coupling can also be assessed spectrophotometrically. The derivatised carrier is used for immunisation by the monoclonal or polyclonal approach using established techniques.

Antibodies are characterised in terms of their reaction with the immunising peptide, other peptides, the carrier molecule, and the protein that is the focus of study. It is critical that the samples containing the authentic cleavage site epitope on the natural protein be available for testing. In addition, peptides that do not have the appropriate terminus are required to demonstrate the terminal specificity. For instance, Vankemmelbeke et $a l^{\beta 6}$ raised an antibody to the sequence Leu-Ala-Gly-GlnArg-Gly-Ile-Val-Gly-Cys, which is a new $\mathrm{N}$-terminal sequence produced after the cleavage of type II collagen by mammalian collagenases. The same peptide, but with an additional Gly at the $\mathrm{N}$-terminus (the amino acid residue normally at this position in the type II collagen molecule), was also synthesised and shown not to react with the cleavage site specific antibody. Antibody characterisation is normally performed by enzyme linked immunosorbent assay (ELISA) inhibition assays, where the immunising peptide is used to coat the plates, and antibody is preincubated with the competing peptide before application to the plate and resolution of the assay. Obviously, the immunising peptide should compete efficiently. If a peptide with an extended or truncated terminus also competes, then the antibody is unlikely to recognize specifically the neoepitope generated from the intact protein. This should be tested directly by using the intact protein as the competing peptide in the inhibition ELISA. If, on the other hand, the immunising peptide is the only one that competes efficiently in the ELISA it is likely to be a cleavage site specific neoepitope antibody.

If the antibody raised is polyclonal, there will invariably be a large quantity of immunoglobulin directed against the carrier molecule. This can be assessed conveniently by western blotting or double immunodiffusion against the unmodified carrier protein. Usually, the presence of these antibodies does not cause a problem, but if for any reason it is decided that they should be removed, this can be done by passage of the antibody preparation through a column derivatised with the carrier molecule. We would recommend coupling the protein to activated $\mathrm{CH}-\mathrm{Sepharose}$ (Amersham/Pharmacia Biotech, Baie d'Urfé, Quebec, Canada), according to the manufacturer's instructions, passing the antibody preparation through the column, and then re-evaluating it. If required, antibodies that have bound to the column can be removed by treatment at low or high $\mathrm{pH}$, although optimal conditions would have to be determined experimentally. Depending on the size and degree of derivatisation of the column, and the quantity of antibody, the preparation should be cleared of antibodies to the carrier protein by one or more passes through the column.

If the antibody is a polyclonal one that reacts not only with the immunising peptide but also with the intact protein or peptide with modified terminus, it might still be possible to adsorb out the unwanted reactivity using a column derivatised with the intact protein, or with the peptide with a modified terminus. Peptides containing a terminal Cys can be conveniently coupled to Sulfolink gel (Pierce Chemicals, Rockford, Illinois, USA) and the resulting resin used for the purification of specific immunoglobulin as outlined above. ${ }^{38} \mathrm{Re}$-evaluation of the selectivity of the purified antibody is then best undertaken by inhibition ELISA.

When they have been thoroughly characterised, cleavage site specific neoepitope antibodies can be used in ELISA, ${ }^{39}$ western blotting, ${ }^{26}$ and immunohistochemistry (fig 5). It should not be forgotten that absolute proof of the occurrence of a specific peptide bond cleavage is still best obtained by $\mathrm{N}$-terminal sequence analysis of the appropriate product, and that this is still the principal way in which such cleavages are first discovered. Therefore, whenever possible, $\mathrm{N}$-terminal sequencing should be performed. This can be done easily after SDS polyacrylamide gel electrophoresis and electroblotting of cleaved proteins to PVDF (polyvinylidine difluoride) membranes, where the resulting bands can be identified by a general protein stain (such as Coomassie brillant blue), and excised for $\mathrm{N}$-terminal sequencing, ${ }^{40}$ or probed using a cleavage site specific neoepitope antibody to the new N-terminus. The use of the same transfer membrane for both processes allows direct comparison of the migration position. ${ }^{41}$

\section{Limitations of the approach}

The detection of protein cleavage products can only take place if these are retained within the biological system for a sufficient length of time. 
It is likely that under many circumstances the products of proteolytic activity are rapidly modified or eliminated from the system by other means. Modification could be achieved simply by the removal of the newly exposed terminal amino acid by the action of an exopeptidase, or it might be the result of the wholesale breakdown of proteins to small peptides or individual amino acids in the lysosome. Rapid removal of cleavage products from the circulation occurs via uptake and clearance, often by endothelial cells or in the liver or kidneys. While it is undoubtedly true that in many instances rapid clearance by removal or modification does occur, in some cases this might just be a widely held assumption rather than a process that has been proved experimentally. By way of illustration, until quite recently the breakdown of aggrecan in cartilage was generally believed to be a wholesale process. This view was based on evidence obtained in vitro, where aggrecan was shown to be highly susceptible to proteolysis by a large number of proteolytic enzymes that cleaved the molecule extensively into a number of differently sized products, which with time were hydrolysed further. It therefore came as a surprise to most people working in the field when it was shown that high $M_{r}$ products could be found in the culture medium of cartilage explants, and similarly sized products with the same N-termini were also present in synovial fluid. $^{42}{ }^{43}$ This discovery has led to a new understanding of the events leading to the loss of aggrecan from cartilage in the development of arthritis. Even in situations where wholesale and apparently indiscriminate breakdown of a protein or proteins is taking place, certain products resistant to further modification may be retained.

Because a particular cleavage product has been shown to exist, it should not be assumed that the pathway leading to this product is necessarily a major pathway. It follows from the argument made above that many products of proteolytic cleavage are not detected easily by cleavage site specific neoepitope antibodies, because of their rapid modification or removal. Therefore, caution should always be used when interpreting the results of experiments with these antibodies, at least until such time as quantitative ELISA has demonstrated the prevalence or otherwise of a particular product. To date, such quantitation has only rarely been carried out, and this is one area in which further progress should be made in the future. On a similar vein, because products of proteolysis must be retained within the biological system, and not rapidly modified, to be readily detectable by the use of cleavage site specific neoepitope antibodies, it follows that the cleavages producing these products may not be rate determining. A rate determining cleavage is one which yields products that are highly susceptible to further modification. An example of this may be the breakdown of fibrillar collagen. The triple helical and fibrillar nature of collagen hinders proteolysis, and it has been generally agreed that the initial cleavage of the molecule produces fragments that are much more susceptible to further breakdown by many proteases. If this were the whole story, the detection of the initial cleavage site should be very difficult. Yet we have seen (fig 5) that it is possible to detect, in vivo, a product resulting from the initial cleavage of the type II collagen molecule. This observation requires us to modify our views on the roles of collagenolytic and gelatinolytic enzymes in collagen breakdown, and demonstrates how the use of cleavage site specific neoepitope antibodies might alter our perceptions of protein modification and breakdown pathways.

\section{Concluding remarks}

Despite the limitations outlined above, cleavage site specific neoepitope antibodies are being used increasingly in a number of fields where selective protein cleavage is important in the maturation, activity, and degradation of proteins, and are aiding our understanding of these processes and how they influence human physiology and pathology. Hopefully, this knowledge will allow the development of new treatment regimens to influence the activity of the proteolytic enzymes responsible. Because of the multitude of circumstances where protein cleavage is important in human health and disease, the potential usefulness of these antibodies is far greater than their current use. Now that the technology has been tried and tested, we anticipate that over the next few years the application of cleavage site specific antibodies will increase rapidly.

The authors wish to thank M Lepik and J Wishart for the illustrations, $M$ Vankemmelbeke for the immunohistochemistry, and trations, $M$ Vankemmelbeke for the

1 Kühn K. The classical collagens: types I, II, and III. In: R Maybe, RE Burgeson, eds. Structure and function of collagen types. San Diego: Academic Press, 1987:1-42.

2 Baumeister W, Cejka Z, Kania M, et al. The proteasome: a macromolecular assembly designed to confine proteolysis macromolecular assembly designed to confine proteoly

3 Haass C, Selkoe DJ. Cellular processing of $\beta$-amyloid Hass C, Selkoe DJ. Cellular processing of $\beta$-amyloid
precursor protein and the genesis of amyloid $\beta$-peptide. Cell precursor protein and

4 Steer ML. How and where does acute pancreatitis begin? Arch Surg 1992;127:1350-3.

5 Couser WG. Pathogenesis of glomerulonephritis. Kidney Int Suppl 1993;42:S19-26.

6 Aznavoorian S, Murphy AN, Stetler-Stevenson WG, et al. Molecular aspects of tumor cell invasion and metastasis. Cancer 1993;71:1368-83.

7 Poole AR. Cartilage in health and disease. In: Koopman WJ, ed. Arthritis and allied conditions: a textbook of rheumatology. Baltimore: Williams and Wilkins, 1997:255-308.

8 Zaidi M, Alam ASMT, Shankar VS, et al. Cellular biology of bone resorption. Biol Rev 1993;68:197-264.

9 Knight KR, Burdon JG, Cook L, et al. The proteinaseantiproteinase theory of emphysema: a speculative analysis of recent advances into the pathogenesis of emphysema. of recent advances into
Respirology 1997;2:91-5.

10 Chapman HA, Riese RJ, Shi GP. Emerging roles for cysteine proteases in human biology. Annu Rev Physiol 1997;59:6388 .

11 Caterson B, Hughes CE, Johnstone B, Mort JS. Immunological markers of cartilage proteoglycan metabolism in animal and human osteoarthritis. In: Kuettner KE, Schleyerbach R, Peyron JG, et al., eds. Articular cartilage and osteoarthritis. New York: Raven Press, 1992:415-27.

2 Weber C, Katayama J, Springer TA. Differential regulation of $\beta 1$ and $\beta 2$ integrin avidity by chemoattractants in eosinophils. Proc Natl Acad Sci USA 1996;93:10939-44.

13 Hollander AP, Heathfield TF, Webber C, et al. Increased damage to type II collagen in osteoarthritic articular carti1722-32.

14 Strickland DK, Steiner JP, Miglioini M, et al. Identification of a monoclonal antibody specific for a neoantigenic determinant on alpha-2-macroglobulin: use for the purification and characterization of binary proteinase-inhibitor com-
plexes. Biochemistry 1988;27:1458-66.

plexes. Biochemistry 1988,27.1458-66.

Young AC, Valadon P, Casadevall A, et al. The threedimensional structures of a polysaccharide binding antibody to Cryptococcus neoformans and its complex with a 
peptide from a phage display library: implications for the identification of peptide mimotopes. F Mol Biol 1997;274: 622-34.

16 Wilson IA, Stanfield RL. Antibody-antigen interactions: new structures and new conformational changes. Curr Opin Struct Biol 1994;4:857-67.

17 Liang H, Fesik SW. Three-dimensional structures of proteins involved in programmed cell death. $7 \mathrm{Mol} \mathrm{Biol}$ 1997;274:291-302.

18 Pacella BJ, Hui KY, Haber E, et al. Induction of fibrin-specific antibodies by immunization with synthetic peptides that correspond to amino termini of thrombin cleavage sites. Mol Immunol 1983;20:521-7.

19 Hui KY, Haber E, Matsueda GR. Monoclonal antibodies to a synthetic fibrin-like peptide bind to human fibrin but not fibrinogen. Science 1983;222:1129-32.

20 Saido TC, Nagao S, Shiramine M, et al. Autolytic transition of mu-calpain upon activation as resolved by antibodies distinguishing between the pre- and post-autolysis forms. $\mathcal{F}$ Bistinguishing between 1992;111:81-6.

21 Kikuchi H, Imajoh-Ohmi S, Kanegasaki S. Novel antibodies specific for proteolyzed forms of protein kinase C: production of anti-peptide antibodies available for in situ analysis of intracellular limited proteolysis. Biochim Biophys Acto 1993; 1162:171-6.

22 Poole AR, Mort JS, Roughley PJ. Methods for evaluating mechanisms of cartilage breakdown. In: Woessner JF Howell DS, eds. Foint cartilage degradation: basic and clinical aspects. New York: Marcel Dekker, 1993:225-60

23 Hughes CE, Caterson B, White RJ, et al. Monoclonal antibodies recognizing protease-generated neoepitopes from cartilage proteoglycan degradation: application to studies of human link protein cleaved by stromelysin. $7 \mathrm{Biol}$ Chem 1992;267:16011-14.

24 Nguyen Q, Liu J, Roughley PJ, et al. Link protein as a monitor in situ of endogenous proteolysis in human articular cartilage. Biochem f 1991;278:143-7.

25 Nguyen O, Murphy G, Hughes CE, et al. Matrix metalloproteinases cleave at two distinct sites on human metalloproteinases cleave at two distinct sites on
cartilage link protein. Biochem $₹$ 1993;295:595-8.

26 Hughes CE, Caterson B, Fosang AJ, et al. Monoclonal antibodies that specifically recognize neoepitope sequences generated by "aggrecanase" and matrix metalloproteinase cleavage of aggrecan: application to catabolism in situ and in vit

27 Lark MW, Williams H, Hoernner LA, et al. Quantitation of a matrix metalloproteinase-generated aggrecan G1 fragment using monospecific anti-peptide serum. Biochem $f$ 1995;307:245-52.

28 Fosang AJ, Last K, Gardiner P, et al. Development of a cleavage-site-specific monoclonal antibody for detecting metalloproteinase-derived aggrecan fragments: detection of fragments in human synovial fluid. Biochem $\mathcal{F}$ 1995;310: 337-43.

29 Lark MW, Bayne EK, Flanagan J, et al. Aggrecan degradation in human cartilage. Evidence for both matrix metalloprotei- nase and aggrecanase activity in normal, osteoarthritic, and rheumatoid joints. $\mathcal{F}$ Clin Invest 1997;100:93-106.

30 Sztrolovics R, Alini M, Roughley PJ, et al. Aggrecan degradation in human intervertebral disc and articular cartilage. Biochem F 1997;326:235-41.

31 Singer II, Scott S, Kawka DW, et al. Aggrecanase and metalloproteinase-specific aggrecan neo-epitopes are induced in the articular cartilage of mice with collagen II-induced arthritis. Osteoarthritis Cartilage 1998;5:407-18.

32 Lee ER, Lamplugh L, Leblond CP, et al. Immunolocalization of the cleavage of the aggrecan core protein at the $\mathrm{Asn}^{341}-\mathrm{Phe}^{342}$ bond, as an indicator of the location of the metalloproteinases active in the lysis of the rat growth plate. Anat Rec 1998;252:117-32.

33 Shingleton WD, Hodges DJ, Brick P, et al. Collagenase: a key enzyme in collagen turnover. Biochem Cell Biol 1996;74:759-75.

34 Martel-Pelletier J, Pelletier JP. Wanted-the collagenase responsible for the destruction of the collagen network in human cartilage! Br f Rheumatol 1996;35:818-20.

35 Billinghurst RC, Dahlberg L, Ionescu M, et al. Enhanced cleavage of type II collagen by collagenases in osteoarthritic articular cartilage. $\mathcal{F}$ Clin Invest 1997;99:1534-45.

36 Vankemmelbeke M, Dekeyser PM, Hollander AP, et al. Characterization of helical cleavages in type II collagen generated by matrixins. Biochem f 1998;330:633-40.

37 Bernatowicz MS, Matsueda GR. Preparation of peptideprotein immunogens using N-succinimidyl bromoacetate as a heterobifunctional crosslinking reagent. Anal Biochem 1986;155:95-102.

38 Roughley PJ, White RJ, Mort JS. The presence of pro-forms of decorin and biglycan in human articular cartilage. Biochem f 1996;318:779-84.

39 Fosang AJ, Last K, Maciewicz RA. Aggrecan is degraded by matrix metalloproteinases in human arthritis. Evidence that matrix metalloproteinases and aggrecanase activities can be independent. F Clin Invest 1996;98:2292-9.

40 Matsudaira P. Sequence from picomole quantities of proteins electroblotted onto polyvinylidene difluoride membranes. F Biol Chem 1987;262:10035-8.

41 Buttle DJ, Fowles A, Ilic MZ, et al. "Aggrecanase" activity is implicated in tumour necrosis factor $\alpha$ mediated cartilage aggrecan breakdown but is not detected by an in vitro assay. 7 Clin Pathol: Mol Pathol 1997;50:153-9.

42 Sandy JD, Neame PJ, Boynton RE, et al. Catabolism of aggrecan in cartilage explants. Identification of a major cleavage site within the interglobular domain. $f$ Biol Chem 1991;266:8683-5.

43 Lohmander LS, Neame PJ, Sandy JD. The structure of aggrecan fragments in human synovial fluid: evidence that aggrecanase mediates cartilage degradation in inflammatory joint disease, joint injury and osteoarthritis. Arthritis Rheum 1993;36:1214-22. 